\title{
The nuclear poly(A) binding protein of mammals, but not of fission yeast, participates in mRNA polyadenylation
}

\author{
UWE KÜHN, JULIANE BUSCHMANN, and ELMAR WAHLE \\ Institute of Biochemistry and Biotechnology, Martin Luther University Halle-Wittenberg, 06099 Halle, Germany
}

\begin{abstract}
The nuclear poly(A) binding protein (PABPN1) has been suggested, on the basis of biochemical evidence, to play a role in mRNA polyadenylation by strongly increasing the processivity of poly(A) polymerase. While experiments in metazoans have tended to support such a role, the results were not unequivocal, and genetic data show that the $S$. pombe ortholog of PABPN1, Pab2, is not involved in mRNA polyadenylation. The specific model in which PABPN1 increases the rate of poly(A) tail elongation has never been examined in vivo. Here, we have used 4-thiouridine pulse-labeling to examine the lengths of newly synthesized poly(A) tails in human cells. Knockdown of PABPN1 strongly reduced the synthesis of full-length tails of $\sim 250$ nucleotides, as predicted from biochemical data. We have also purified $S$. pombe Pab2 and the $S$. pombe poly(A) polymerase, Pla1, and examined their in vitro activities. Whereas PABPN1 strongly increases the activity of its cognate poly(A) polymerase in vitro, Pab2 was unable to stimulate Pla1 to any significant extent. Thus, in vitro and in vivo data are consistent in supporting a role of PABPN1 but not $S$. pombe Pab2 in the polyadenylation of mRNA precursors.
\end{abstract}

Keywords: poly(A) binding protein; poly(A) polymerase; mRNA polyadenylation; pre-mRNA 3'; processing

\section{INTRODUCTION}

The poly(A) tails of eukaryotic mRNAs are covered by specific proteins, which differ between the nucleus and the cytoplasm. The cytoplasmic poly(A) binding proteins are composed of four RRM domains and a C-terminal $\alpha$-helical domain and function mainly in translation and the control of mRNA decay (Mangus et al. 2003; Kühn and Wahle 2004; Wigington et al. 2014). The nuclear poly(A) binding proteins (PABPN1 in mammals) contain a single RRM domain, which mediates RNA binding together with a presumably unstructured C-terminal domain rich in dimethylarginine. An N-terminal highly acidic region of unknown function is less conserved and separated from the RRM by a coiled-coil segment (Nemeth et al. 1995; Smith et al. 1999; Kühn et al. 2003; Ge et al. 2008; Song et al. 2008). Apparently with the exception of S. cerevisiae (Winstall et al. 2000), PABPN1 appears to be conserved among all model organisms. Additional RNA binding proteins with specificity for poly(A) have been reviewed (Wigington et al. 2014).

PABPN1 was discovered through its stimulating influence on the polyadenylation of mRNA precursors in vitro (Wahle 1991a). Biochemical experiments led to the following model: After cleavage of the pre-mRNA, polyadenylation is initiated

Corresponding authors: ukuehn@biochemtech.uni-halle.de, ewahle@ biochemtech.uni-halle.de

Article is online at http://www.rnajournal.org/cgi/doi/10.1261/rna.057026. 116. by poly $(A)$ polymerase with the help of the cleavage and polyadenylation specificity factor (CPSF), which binds the polyadenylation signal AAUAAA. The polymerase by itself is barely active due to a low affinity for RNA, but acts more efficiently in the presence of CPSF. PABPN1 associates with the growing poly(A) tail once it is 10-12-nt long and contributes to the stimulation of poly(A) polymerase: CPSF and PABPN1 cooperatively tether the enzyme to the RNA and thus make it highly processive. The processive reaction generates a poly $(\mathrm{A})$ tail of $250 \mathrm{nt}$, then the contact between CPSF and the advancing poly $(\mathrm{A})$ polymerase is severed. During further extension of the tail, poly(A) polymerase is stimulated only by PABPN1, thus poorly processive and slow. This mechanism results in relatively homogeneous polyadenylation products near 250 nt in length (Wahle 1995; Kerwitz et al. 2003; Kühn et al. 2009). Both rapid elongation and the final product length are very similar to what has been observed in early pulse-labeling studies in vivo (Sawicki et al. 1977; Brawerman 1981). In agreement with a function in pre-mRNA polyadenylation in the cell nucleus, mammalian PABPN1 is nuclear at steady state (Krause et al. 1994; Calado et al. 2000).

PABPN1 has functions in addition to nuclear poly(A) tail elongation. A participation in cytoplasmic polyadenylation

(C) 2017 Kühn et al. This article is distributed exclusively by the RNA Society for the first 12 months after the full-issue publication date (see http:// rnajournal.cshlp.org/site/misc/terms.xhtml). After 12 months, it is available under a Creative Commons License (Attribution-NonCommercial 4.0 International), as described at http://creativecommons.org/licenses/by-nc/4.0/. 
during embryo development has been described for PABP2, the PABPN1 ortholog of Drosophila (Benoit et al. 2005), and an embryo-specific ortholog in Xenopus is also localized in the cytoplasm (Cosson et al. 2004; Good et al. 2004). PABPN1 affects pre-mRNA cleavage at the poly(A) site, including alternative poly(A) site choice (de Klerk et al. 2012; Jenal et al. 2012; Chartier et al. 2015; Li et al. 2015), in agreement with its observed association with nascent RNA (Bear et al. 2003; Lemieux and Bachand 2009). Studies in S. pombe, in which the gene encoding the ortholog of PABPN1, pab2, is not essential (Perreault et al. 2007), first indicated a role of the protein in $3^{\prime}$ trimming or degradation of RNA: Cells with a $\triangle p a b 2$ mutation accumulate polyadenylated, $3^{\prime}$-extended snoRNA precursors. This has been attributed to Pab2 promoting the trimming of such RNAs by the exosome (Lemay et al. 2010). Pab2-dependent exosome activity was then found to be responsible for the degradation of meiosis-specific transcripts (St-Andre et al. 2010; Yamanaka et al. 2010) and of unspliced pre-mRNAs in S. pombe (Lemieux et al. 2011) and of a number of lncRNAs and other RNAs in human cells (Beaulieu et al. 2012; Bresson and Conrad 2013; Bresson et al. 2015). A knockdown of PABPN1 in mammalian cells also leads to an accumulation of polyadenylated telomerase RNA precursors, reflecting a role of the protein in polyadenylation of these RNAs and/ or in their processing by the $3^{\prime}$ exonuclease PARN (Nguyen et al. 2015).

A role of PABPN1 in pre-mRNA polyadenylation has been supported by a number of in vivo studies, but the results were not unequivocal. In Drosophila, the PABP2 gene is essential for viability: Flies homozygous for a null allele die at late embryonic/early larval stages even before maternally supplied Pabp2 protein has been exhausted. Bulk poly(A) and steady-state poly(A) tails of two specific messages were found to be shorter in pabp2 mutants; however, poly(A) tails of a third mRNA, Hsp70, were not affected. Amino acids in the coiled-coil domain that are essential for the ability of PABPN1 to stimulate poly(A) polymerase were originally identified in the mammalian protein. The equivalent residues are also essential for the function of fly Pabp2 in vivo. These results support an essential role of PABP2 in pre-mRNA polyadenylation (Benoit et al. 2005).

Regarding mammalian cells, knockdown of PABPN1 in myoblasts led to a modest decrease in steady-state bulk poly(A) tail length (Apponi et al. 2010). Splicing of some 3'terminal exons is dependent on polyadenylation, and these splicing events are sensitive to a depletion of either PABPN1 or canonical poly(A) polymerase, as would be expected from the cooperation of these proteins in poly(A) tail synthesis (Muniz et al. 2015). Nuclear degradation of different types of transcripts by the exosome depends on their "hyperadenylation," i. e., addition of excessively long poly(A) tails (Bresson and Conrad 2013; Bresson et al. 2015). RNAi and experiments with a dominant-negative mutant showed that hyperadenylation depends on the "canonical" poly(A) polymerases $\alpha$ and $\gamma$ and on PABPN1, again consistent with PABPN1 stimulating the activity of poly(A) polymerase. The addition of "regular" poly(A) tails was not prevented either by knockdown of PABPN1 or by the combined depletion of both canonical poly(A) polymerases, suggesting that low concentrations of the proteins involved are sufficient for regular polyadenylation but not hyperadenylation. As discussed by the authors (Bresson et al. 2015), this can be explained by the model described above: Distributive hyperadenylation depends only on poly(A) polymerase and PABPN1, whereas processive polyadenylation generating regular poly(A) tails is also supported by CPSF and thus is more robust.

In contrast to these studies in metazoan cells, experiments examining S. pombe $\triangle p a b 2$ mutants provide the most persuasive argument against a conserved role of PABPN1 in nuclear pre-mRNA polyadenylation: Bulk poly(A) is longer in the mutant than in the wild-type, reflecting the presence of some hyperadenylated RNA species. Poly(A) tail lengths of two specific mRNAs analyzed did not differ between mutant and wild-type (Perreault et al. 2007; Lemay et al. 2010). These data suggest that either a function of PABPN1 in pre-mRNA polyadenylation is not conserved in S. pombe or that the data supporting such a function in mammalian cells have been misleading.

Experiments concerning the role of PABPN1 in polyadenylation in vivo have had to deal with two problems: First, with the exception of the viable $S$. pombe $\Delta p a b 2$ mutant, studies are inevitably limited to situations in which some residual PABPN1 is still present, and even low concentrations of the protein have a significant effect on polyadenylation in vitro (Wahle 1995). Second, the extent to which a depletion of PABPN1 can be expected to cause a detectable phenotype in the steady-state length distribution of mRNA poly(A) tails is unclear: Even a complete PABPN1 depletion would not be expected to result in an outright failure of polyadenylation, as pre-mRNAs will still be polyadenylated in a less rapid manner due to the CPSF-dependent activity of poly(A) polymerase. The tail lengths generated in this type of reaction would depend on many variables, e.g., the time available before nuclear export of the RNA. In addition, differences in poly(A) tail lengths initially generated in the cell nucleus can be expected to be blurred by subsequent poly(A) tail shortening in the cytoplasm. For the same reasons, unaltered steady-state levels of mRNAs upon PABPN1 depletion (Beaulieu et al. 2012) are not a convincing argument against a role of PABPN1 in polyadenylation.

Here we confirm that efficient depletion of PABPN1 has a modest effect on steady-state lengths of bulk poly(A) in a mammalian cell line. In contrast, the inhibition of initial poly(A) synthesis was much more pronounced. We also report that the $S$. pombe Pab2 protein does not stimulate its cognate poly(A) polymerase to a significant extent in vitro, consistent with genetic data. The data thus support a role of mammalian PABPN1 in pre-mRNA polyadenylation but suggest that this function is not universally conserved. 
A

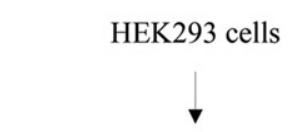

1st ctrl/ PABPN1 knock down

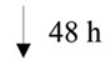

2nd ctrl/ PABPN1 knock down

> $48 \mathrm{~h}$

4-thiouridine

จ 10 or 30 min. pulse

isolate total RNA (Trizol)

$\checkmark$ Biotin-HPDP

biotinylated RNA

$\checkmark$ Streptavidin beads

bound RNA= flow through= steady state RNA new RNA

radioactive 3' labeling of RNA

RNases A/T1 digestion

8.3 M urea PAGE

D
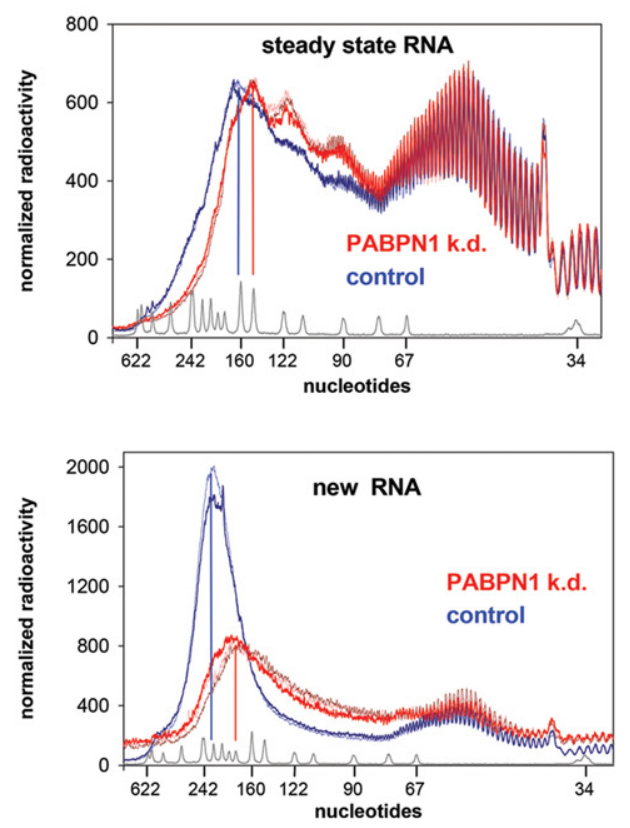

B
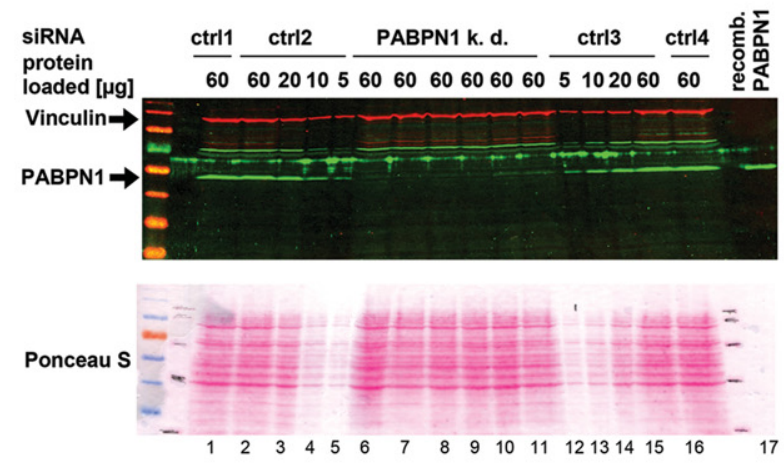

C

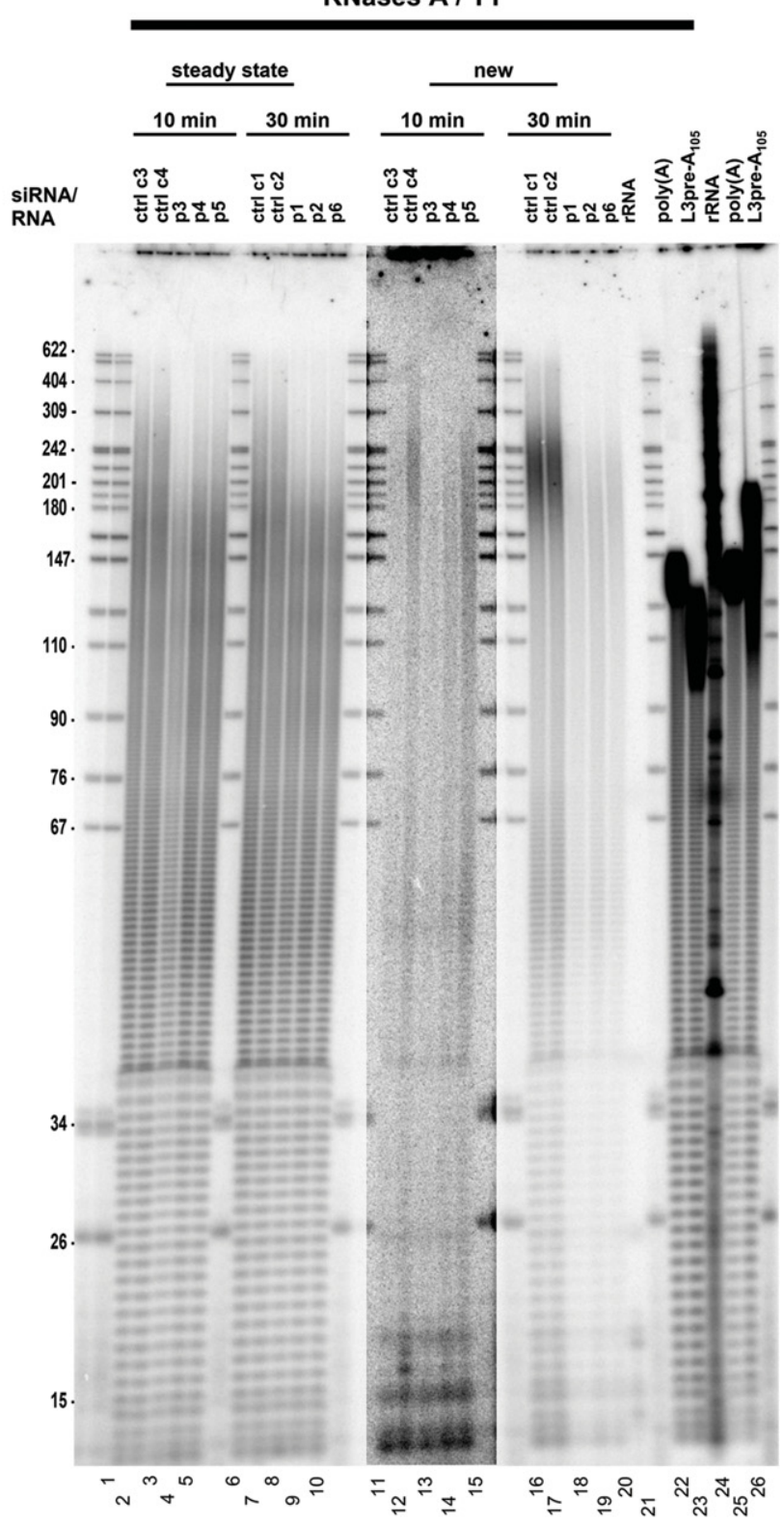

FIGURE 1. (Legend on next page) 


\section{RESULTS}

\section{Mammalian PABPN1 is involved in poly(A) tail synthesis in vivo}

In order to specifically address the idea that PABPN1 affects the rate of poly $(\mathrm{A})$ tail elongation, we designed an experiment to examine the lengths of newly synthesized poly(A) tails (Fig. 1A): HEK293 cells were treated twice with siRNAs directed against PABPN1 or with a control siRNA. Western blots showed that the siRNAs against PABPN1 caused a depletion of the protein by more than 90\% (Fig. 1B). The cells were pulse-labeled with 4-thiouridine for 10 or $30 \mathrm{~min}$. After isolation of total RNA, pulse-labeled RNA was biotinylated and separated from unlabeled steady-state RNA on streptavidin beads. In both pulse-labeled and steady-state RNA, bulk poly $(\mathrm{A})$ tail lengths were then measured by $3^{\prime}$-end-labeling of the RNA and digestion of all non-poly $(\mathrm{A})$ sequences by a mixture of RNases A and T1. The remaining end-labeled poly(A) tails were analyzed on denaturing polyacrylamide gels (Fig. 1C,D). Controls showed that the nuclease treatment left poly(A) intact but degraded other sequences to completion (Fig. 1C, lanes 21-26). In four pulse-labeled control samples (four cell samples transfected with control siRNA in parallel but independently, two each for the 10-min and 30min pulse), a pronounced peak was visible at a poly(A) tail length between 200 and $240 \mathrm{nt}$ (Fig. 1C, lanes 11,12,16, and 17 and Fig. 1D). This is in excellent agreement with early radioactive pulse-labeling studies leading to an estimate of an average length of $230 \mathrm{nt}$ for newly synthesized poly(A) tails (Brawerman 1981). In the corresponding samples of the PABPN1 knockdown (six samples transfected in parallel but independently, three each for the 10-min and 30-min pulse), two differences were obvious in comparison to the controls: The length distribution of poly(A) tails was much more uniform with only a weak peak at the upper limit. In addition, the poly(A) tails forming this weak peak were up to $50 \mathrm{nt}$ shorter compared to the main peak of the control samples (Fig. 1C, lanes 13-15 and 18-20 and Fig. 1D). Even in the steady-state samples a clear size difference was visible, with poly(A) tails in the intensity peak $\sim 20 \mathrm{nt}$ and those at the upper end of the length distribution 20-80 nt shorter in the PABPN1 knock- down (Fig. 1C, lanes 1-10 and Fig. 1D). Similar results were obtained with an independent siRNA directed against PABPN1 (Supplemental Fig. 1). A deficit in the synthesis of full-length poly(A) tails upon PABPN1 depletion was also visible by RT-PCR analysis of two individual mRNAs (Supplemental Fig. 2). The results fully support the model for PABPN1 activity based on in vitro studies: In normal cells, the cooperative stimulation of poly $(\mathrm{A})$ polymerase by CPSF and PABPN1 leads to a rapid poly(A) tail elongation to 200-250 nucleotides (nt). At this length, CPSF loses its contact with poly(A) polymerase so that only a poorly processive and therefore slow elongation beyond $\sim 250 \mathrm{nt}$ is possible. This results in the sharp peak in the length distribution of newly synthesized tails visible in the control samples. Upon PABPN1 depletion, polyadenylation is dominated by a poorly processive, inefficient reaction relying on CPSF alone, in which poly(A) tails grow more slowly without a clear cut-off.

Not surprisingly, cell growth was disturbed by the knockdown of PABPN1. For example, total RNA yield in the PABPN1 knockdown samples was $73 \%$ of the control in the experiment of Figure $1(90 \pm 15 \mu \mathrm{g}$ RNA in the control, $n=4 ; 65 \pm 12 \mu \mathrm{g}$ RNA in the PABPN1 knockdown, $n=6$ ). In order to ascertain that the observed effects on poly(A) synthesis were specific and not due to generally poor conditions of the cells, we carried out two types of controls: First, nucleotide pools in PABPN1 knockdown and control cells were measured by HPLC. Pools of AMP, ADP, and ATP were indistinguishable in PABPN1-depleted and control cells, and, as a result, the energy charge was also the same (Fig. 2A,B). This indicates a normal metabolic state of the knockdown cells and, in particular, excludes low ATP levels as a cause for inefficient polyadenylation. As a second control, the efficiencies of splicing and $3^{\prime}$ cleavage of two different premRNAs were examined by RT-qPCR analysis of the newly made RNA (Fig. 2C-E). Amounts of unprocessed precursor RNAs were normalized against the corresponding mature RNAs (GAPDH and ACTB) as a gauge of processing efficiency. No significant difference was found between PABPN1-depleted and control cells. Comparison to the levels of the stable U6 snRNA did not reveal an accumulation of unprocessed precursor RNAs either (Fig. 2C-E). We conclude that the defect in pre-mRNA polyadenylation observed upon

FIGURE 1. Depletion of PABPN1 by RNAi inhibits the synthesis of poly(A) tails. (A) Overview of the procedure to examine the effect of PABPN1 depletion on poly(A) tail synthesis in vivo. Biotin-HPDP is the reagent used for biotinylation of 4sU-labeled RNA. (B) A PABPN1 knockdown was performed with siRNA PABPN1_b in six cell samples in parallel. The Western blot (upper panel) shows that, in all samples, PABPN1 content was reduced to $10 \%$ or less in comparison to the controls (four independent samples). Recombinant PABPN1 was used as a positive control (lane 17). The first, second, and second to last lanes contained two different sets of marker proteins, one of which cross-reacted with the antibody. In addition to Ponceau staining of the membrane (lower panel), vinculin and a band cross-reacting with the PABPN1 antibody were used as loading controls. (C) Result of the analysis carried out as outlined in A. (P1-P6) RNA preparations obtained from PABPN1 knockdown cells. Lanes 11-15 are longer exposures from the same gel. Three controls for the specificity of the RNase digestion were carried out: Commercial ribosomal RNA from E. coli was digested to completion (compare lanes 21 and 24), size-fractionated poly(A) was not affected (compare lanes 22 and 25), and L3pre RNA with a poly(A) tail of $\sim 100 \mathrm{nt}$ was reduced to the expected size (compare lanes 23 and 26). Sizes of DNA markers, loaded in multiple lanes, are indicated on the left. (D) Lanes 6-10 (upper panel) and 16-20 (lower panel) from the gel shown in $C$ were scanned. Knockdown samples are shown in different shades of red, control samples are in shades of blue. Vertical lines show the positions of the intensity peaks compared to the size markers. This is one out of four experiments performed with the same siRNA (in total, 13 knockdown samples each for control and PABPN1 siRNA). 
A
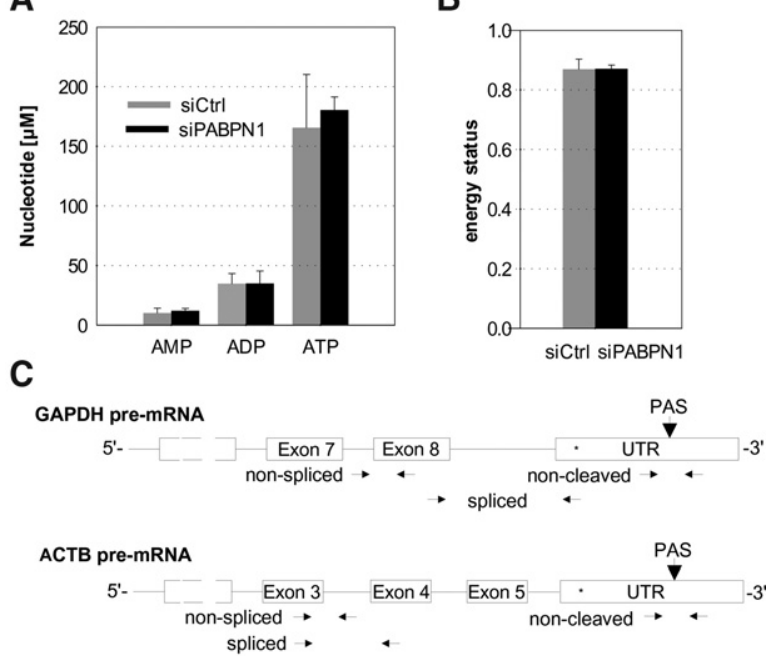

D

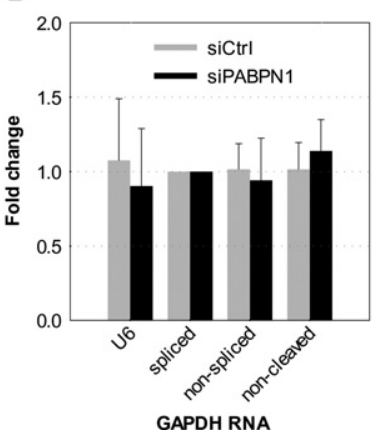

E

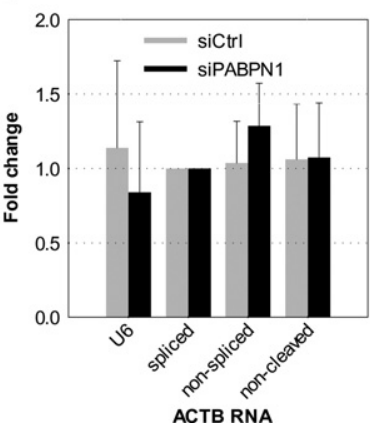

FIGURE 2. Knockdown of PABPN1 does not cause ATP depletion or general defects in RNA processing. (A) Knockdown of PABPN1 and controls were carried out. Nucleotide pools were analyzed by HPLC and the concentrations of adenine nucleotides determined (see Materials and Methods). Note that, for technical reasons, these analyses were not carried out on the same cells that were used for the analyses shown in Figure 1. However, the extent of PABPN1 depletion was similar to that shown in Figure 1B. $(B)$ The energy charge was calculated from the data in $A$. Data in $A$ and $B$ were averaged from three experiments carried out in parallel but independently. Analysis of an additional set of samples showed similar results. (C) Scheme showing the relevant parts of the gene structures of GAPDH and actin $\beta$ and the locations of primers used for PCR. The asterisks indicate the positions of the stop codons. (PAS) poly(A) site. $(D, E)$ Random priming was used for cDNA synthesis from the 4sU-pulse-labeled samples of an experiment as in Figure $1(n=4$ each for PABPN1 knockdown samples and controls), and $\mathrm{qPCR}$ was performed with the primer combinations shown in C. Data for nonspliced and noncleaved mRNA precursors were normalized to the levels of the corresponding spliced RNAs. U6 RNA was amplified as a control and was also normalized to mature GAPDH RNA $(D)$ or ACTB RNA $(E)$.

depletion of PABPN1 is specific and reflects the role of the protein in enhancing the activity of poly(A) polymerase.

\section{The Schizosaccharomyces pombe ortholog of PABPN1 does not affect its cognate poly(A) polymerase in vitro}

While the results described above lend satisfactory support to the model of PABPN1 function derived from biochemical ex- periments, they leave open the question why a $\Delta p a b 2$ mutation in $S$. pombe does not cause a defect in polyadenylation. PABPN1 strongly stimulates the activity of mammalian poly (A) polymerase in vitro, either in a cooperative reaction with CPSF or on its own (Wahle 1991a; Kerwitz et al. 2003; Kühn et al. 2009). Therefore, we examined whether S. pombe Pab2 would have a comparable effect on its cognate poly(A) polymerase.

For the purpose of these experiments, both Pab2 and Pla1, the canonical poly(A) polymerase from $S$. pombe (Ohnacker et al. 1996) were overproduced in E. coli and purified (Fig. 3A). In nitrocellulose filter binding experiments, purified S. pombe Pab2 bound $\mathrm{A}_{14}$ with an apparent $K_{\mathrm{D}}$ of $5.3 \pm$ $1.1 \mathrm{nM}(n=3)$ (Fig. 3B), very similar to the affinity determined for mammalian PABPN1 (2-5 nM under the conditions used here; $8 \mathrm{nM}$ under the conditions used previously [Kühn et al. 2003]). Binding of Pab2 and PABPN1 to $\mathrm{A}_{100}$ differed by less than twofold (Fig. 3C). In electrophoretic mobility shift assays, increasing amounts of Pab2 formed a ladder of retarded complexes with $\mathrm{A}_{100}$ (Fig. 3D), again in good agreement with the behavior of PABPN1 (Kühn et al. 2003). In filter-binding competition assays, $\mathrm{Pab} 2$ proved to be specific for poly(A), in agreement with earlier pull-down assays (Perreault et al. 2007), but it was less specific than PABPN1; tRNA in particular was a surprisingly efficient competitor (Fig. 3E). The activity of Plal was tested by an assay that measures the elongation of $\mathrm{A}_{14}$ primers with $\left[\alpha^{3}{ }^{32} \mathrm{P}\right]$-labeled ATP in the presence of $\mathrm{Mn}^{2+}$ by adsorption of the polymeric reaction product to DEAE paper and scintillation counting (see Materials and Methods). The activity of Pla1 was comparable to the activities of mammalian poly(A) polymerase and S. cerevisiae Pap1. For example, under the reaction conditions of Lingner et al. (1991), specific activities were $5.7 \mu \mathrm{mol}$ AMP incorporated $/ \mathrm{mg} / \mathrm{min}$ for Pla1, $3.2 \mu \mathrm{mol} \mathrm{AMP} / \mathrm{mg} / \mathrm{min}$ for bovine poly(A) polymerase, and $\sim 5.5 \mu \mathrm{mol} \mathrm{AMP} / \mathrm{mg} / \mathrm{min}$ for $S$. cerevisiae Pap1; specific activities were approximately twofold higher but again similar under the conditions of Wahle (1991b). On the basis of these experiments, we conclude that purified S. pombe Pab2 and Pla1 were functional proteins suitable for further biochemical studies.

Effects of poly(A) binding proteins on poly(A) polymerase activity were tested in assays in which the primer-dependent incorporation of labeled AMP was measured in the presence of $\mathrm{Mg}^{2+}$. Under these conditions, the activity of poly(A) polymerase by itself is weak and stimulatory activities are revealed (Wahle 1991b and references cited therein). As a positive control, PABPN1 stimulated the activity of its cognate poly(A) polymerase by more than 40 -fold, in agreement with published studies (Kerwitz et al. 2003). In contrast, stimulation of Pla1 by Pab2 did not exceed a factor of $\sim 3$ (Fig. 4A). As heterologous combinations of poly(A) binding protein and poly(A) polymerase permitted a slightly more vigorous stimulation, we consider these effects insignificant and very likely nonspecific. Similar effects were seen when the poly(A) primer was titrated either by itself or as a complex with PABPN1 or 

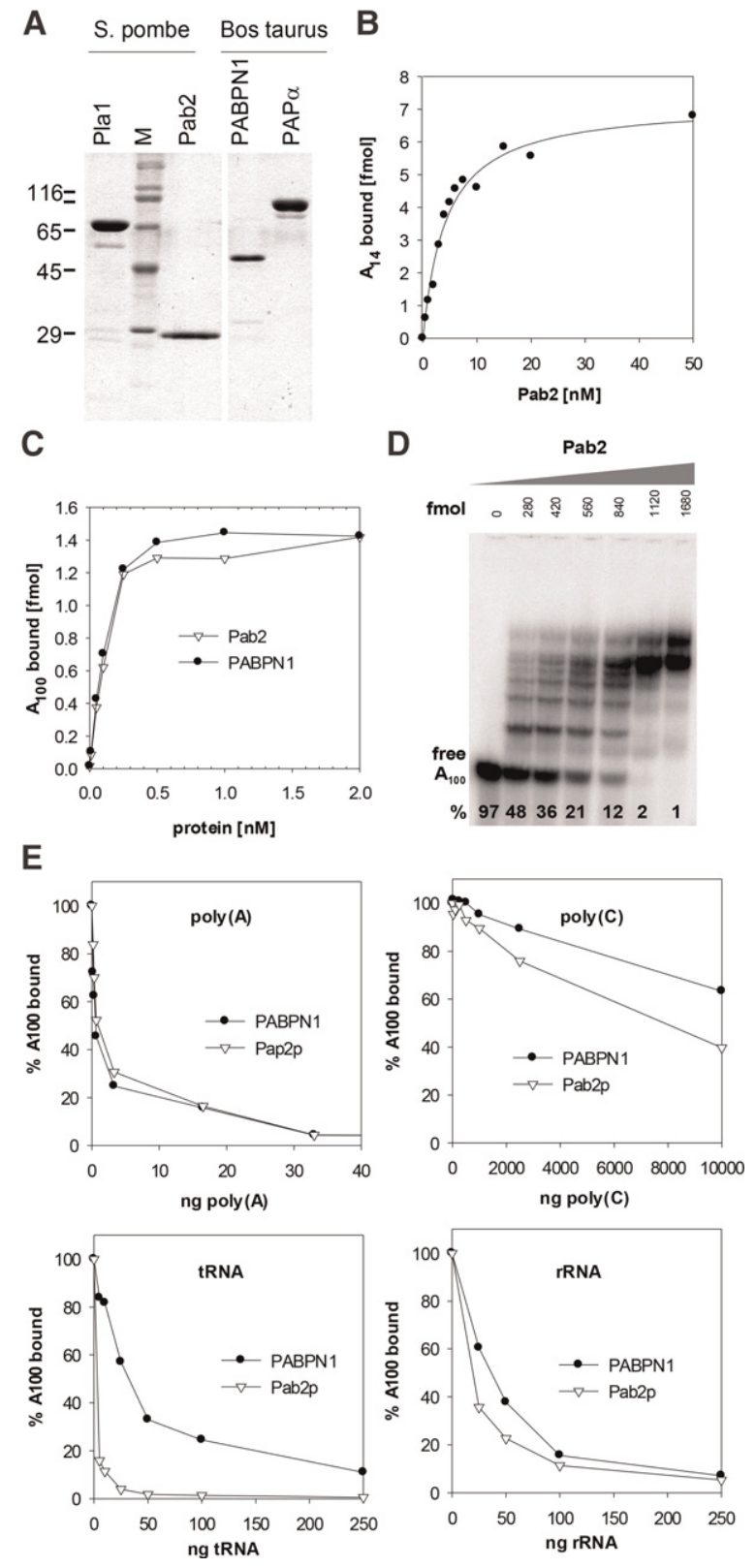

FIGURE 3. Purified S. pombe Pab2 and Pla1 are functional in vitro. $(A)$ Coomassie-stained SDS-polyacrylamide gel displaying the purified proteins used for the biochemical assays. $(B)$ A filter-binding experiment was carried out with $S$. pombe $\mathrm{Pab} 2$ and radiolabelled $\mathrm{A}_{14}$. The line represents a fit to a binary binding equilibrium. One representative experiment of three is shown. $(C)$ Filter-binding experiments were carried out with $S$. pombe Pab2 and bovine PABPN1 and labeled $\mathrm{A}_{100}$. The curves connect the data points and were not fitted. $(D)$ Complexes between $S$. pombe $\mathrm{Pab} 2$ and $80 \mathrm{fmol}$ labeled $\mathrm{A}_{100}$ were analyzed by nondenaturing gel electrophoresis. $(E)$ Complexes between $S$. pombe Pab2 or bovine PABPN1 and labeled $A_{100}$ were challenged by increasing concentrations of unlabeled poly(A), poly (C), tRNA, and E. coli rRNA as competitors as indicated. Note that the scales on the $x$-axes are different. Experiments in $C-E$ were carried out at least twice with similar results.

Pab2: PABPN1 stimulated the activity of mammalian poly(A) polymerase strongly by lowering the $K_{\mathrm{M}}$ as reported previously (Kerwitz et al. 2003). In contrast, the $K_{\mathrm{M}}$ of Pla2 for the poly(A) primer was not affected by Pab2 (Fig. 4B,C). Thus, Pab2 is lacking a productive interaction with its cognate poly(A) polymerase and does not affect the activity of the enzyme.

\section{DISCUSSION}

A role of PABPN1 in increasing the rate of poly(A) tail elongation and poly(A) tail length control in the cell nucleus had been proposed on the basis of biochemical studies with the mammalian protein. However, this function of the protein had never been specifically examined in vivo. We now report that siRNA-mediated knockdown of PABPN1 in mammalian cells and pulse-labeling of newly synthesized RNA revealed the effect predicted by in vitro data, namely a strong reduction in full-length poly(A) tails and, consequently, a more heterogeneous size distribution and shorter average length in comparison to control cells. We conclude that the activities of PABPN1 in the test tube reflect the protein's function in mRNA polyadenylation in the living cell.

The results of our pulse-labeling assays, showing a pronounced peak between 200 and $240 \mathrm{nt}$ for newly synthesized poly(A) tails, are in full agreement not only with previous bulk measurements based on radioactive pulse-labeling (Brawerman 1981), but also with analyses of numerous individual RNAs: From Table 1 in Chen and Shyu (1994), initial tail lengths between 179 and $239 \mathrm{nt}$ can be calculated for 23 different mRNAs, with an average of $205 \mathrm{nt}$, and initial tail lengths around $200 \mathrm{nt}$ were also reported for several other mRNAs (Shyu et al. 1991; Schiavi et al. 1994). Recent transcriptome-wide analyses of steady-state poly(A) tail lengths (Chang et al. 2014; Subtelny et al. 2014) showed an upper limit of tail lengths between 200 and $250 \mathrm{nt}$, although technical limitations in the analysis of very long tails may have contributed to this.

In contrast to newly synthesized RNAs, steady-state RNAs have a very heterogeneous poly $(\mathrm{A})$ tail length distribution, which is due to gradual shortening while the mRNAs are being translated in the cytoplasm. Transcriptome-wide studies reported median poly(A) tail lengths between 50 and $100 \mathrm{nt}$ in various mammalian cell lines (Chang et al. 2014; Subtelny et al. 2014). Our analysis of steady-state RNA (Fig. 1) is generally consistent with these data, but appears to show a higher proportion of longer tails. Whether this is due to differences in the biological material or the analysis method is unclear.

In contrast to PABPN1, the function of the $S$. pombe ortholog of PABPN1, Pab2, had so far only been investigated genetically; the results did not support a role in nuclear mRNA polyadenylation. This article reports that Pab2 binds specifically and with high affinity to poly(A), very similar to its mammalian cousin. However, whereas PABPN1 is able to increase the activity of mammalian poly(A) polymerase by lowering the $K_{\mathrm{M}}$, i.e., increasing the apparent affinity of the enzyme for the primer RNA (Fig. 4; Kerwitz et al. 2003), Pab2 did not stimulate the activity of the S. pombe poly(A) polymerase, Pla1, to a significant extent and had 

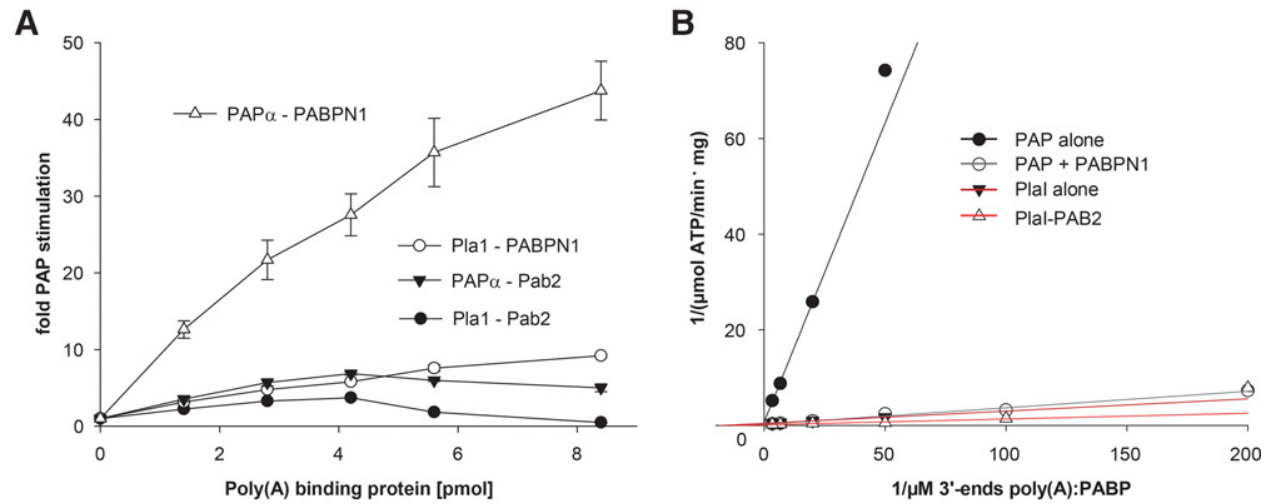

\begin{tabular}{|c|c|c|c|}
\hline Polymerase & Substrate & $\mathrm{K}_{\mathrm{M}}[\mathrm{nM}]$ & $\begin{array}{c}v_{\max } \\
{[\mu \mathrm{mol} \text { ATP/min } \mathrm{mg}]}\end{array}$ \\
\hline \multirow[t]{2}{*}{$\mathrm{PAP} \alpha$} & $\mathrm{A}_{45}$ & 1610 & 1.3 \\
\hline & PABPN1:A45 & 107 & 3.2 \\
\hline \multirow[t]{2}{*}{ Plal } & $A_{45}$ & 59 & 2.2 \\
\hline & $\mathrm{Pab} 2: \mathrm{A}_{45}$ & 76 & 6.3 \\
\hline
\end{tabular}

FIGURE 4. S. pombe Pab2 does not stimulate its cognate poly(A) polymerase. (A) The activity of poly(A) polymerases (S. pombe Pla1 or bovine PAP) was measured in the presence of different amounts of $S$. pombe Pab2 or bovine PABPN1 as described in Materials and Methods. Data are averaged from two independent experiments. Error bars indicate the spread of the data and are too small to be visible for most data points in the three bottom curves. Note that the amount of PABPN1 required for the stimulation of bovine poly(A) polymerase is higher than expected from its ratio to the poly (A) primer used mostly because the amount of poly(A) increases up to about sixfold in the course of the reaction. (B) The influence of the cognate nuclear poly $(\mathrm{A})$ binding proteins on the steady-state kinetic parameters of $\mathrm{PAP} \alpha$ and $\mathrm{PlaI}$ was determined. The poly $(\mathrm{A})$ primer $\left(\mathrm{A}_{45}\right)$ was titrated either by itself (20-300 nM 3' ends) or together with either PABPN1 or Pab2 in amounts sufficient to coat the RNA (5-300 nM 3' ends with 15-900 nM protein). Reaction mixtures containing $20-200 \mathrm{fmol}$ of PAP $\alpha$ or Pla1, as indicated, were assembled on ice, preincubated for $10 \mathrm{~min}$ at $32^{\circ} \mathrm{C}$, and the reactions were started by the addition of radioactive-labeled ATP. After incubation for 10-30 min, they were stopped by the addition of $5 \mathrm{mM}$ EDTA, and AMP incorporation was determined by DEAE absorption (see Materials and Methods). Data are shown as a double-reciprocal plot. (C) Steadystate kinetic parameters derived from $B$.

no effect on the enzyme's $K_{\mathrm{M}}$ for the primer RNA. This is in agreement with the genetic data showing that mRNA polyadenylation in $S$. pombe is not inhibited by the absence of Pab2 (Perreault et al. 2007; Lemay et al. 2010).

In conclusion, in vitro and in vivo data are congruent for both mammalian PABPN1 and its S. pombe ortholog, Pab2, supporting a role in pre-mRNA polyadenylation for the former but not the latter. Thus, even though PABPN1 and its orthologs are widely conserved in evolution, this particular function has been either lost in S. pombe and perhaps other organisms or acquired later in evolution.

\section{MATERIALS AND METHODS}

\section{Cell culture, RNAi, and metabolic labeling of RNA}

HEK293 cells were grown in DMEM GlutaMax (Invitrogen) supplemented with 10\% FCS (FCS Superior, Biochrom KG) and 1\% penicillin/streptomycin solution (Invitrogen) at $37^{\circ} \mathrm{C}$ with $5 \%$ $\mathrm{CO}_{2}$. Cells were split into six-well plates and transfected with 50 pmol siRNAs and $5 \mu \mathrm{L}$ of Lipofectamine RNAiMax (Invitrogen) per well 1-3 d later, depending on their initial density, at $50 \%-$ 90\% confluency (day 1). The following siRNAs were used: siCtrl (UGGGCGUCGUGGAGGCUUUTT, Eurofins MWG Operon),
siPABPN1_b (GGAGCUACAGAACGAGGUATT, Eurofins MWG Operon), and siPABPN1_8 (CCCAAAGGGUUUGCGUAUAUA, Qiagen \#SI04763577). After 4-6 h incubation, the cells were split to three or four wells. On day 3 , the siRNA treatment was repeated. Cells derived from one original well were combined and spread on a 6- $\mathrm{cm}$ or $10-\mathrm{cm}$ dish for protein or RNA preparation, respectively. For 4-thiouridine (4-sU) labeling, the medium was renewed on day 4. On day 5 ( $\sim 96 \mathrm{~h}$ after the first transfection), cells were treated for 10 or $30 \mathrm{~min}$ with $500 \mu \mathrm{M}$ 4-thiouridine (Sigma-Aldrich) or not. Total RNA was isolated by a modified TRIzol method (Chomczynski and Mackey 1995).

\section{RNA analyses}

The biotinylation and purification of 4-thiouridine-labeled RNA was done as previously described (Dolken et al. 2008) with 50$100 \mu \mathrm{g}$ total RNA.

For poly(A) tail length determination, $\sim 1.5 \mu \mathrm{g}$ total RNA or $25 \%$ of one batch of purified 4sU-labeled RNA was $3^{\prime}$ end-labeled in $20 \mu \mathrm{L}$ buffer $(20 \mathrm{mM}$ Tris-Cl, pH 7.0, $50 \mathrm{mM} \mathrm{KCl}, 1.4 \mathrm{mM}$ $\mathrm{MnCl}_{2}, 10 \%$ glycerol, $40 \mathrm{mg} / \mathrm{L}$ methylated BSA, $1 \mathrm{mM} \mathrm{DTT}$ ) containing $300 \mathrm{ng}$ recombinant $S$. cerevisiae poly(A) polymerase, $40 \mathrm{U}$ Ribolock RNase inhibitor (Fermentas/Thermo Scientific), and 5 $\mu \mathrm{Ci}\left[\mathrm{a}^{32} \mathrm{P}\right]$-cordycepintriphosphate (PerkinElmer) for $1 \mathrm{~h}$ at $30^{\circ} \mathrm{C}$. For control purposes, $1.5 \mu \mathrm{g}$ E. coli rRNA (Roche), 2 pmol 
$3^{\prime}$ ends size-fractionated poly(A) ( 120-150 nt), and 100 fmol L3pre- $\mathrm{A}_{105}$ (Kerwitz et al. 2003) were also 3' labeled. Labeling was stopped by incubation with $10 \mu \mathrm{g}$ proteinase $\mathrm{K}$ and $10 \mu \mathrm{g}$ glycogen (Roche) in stop-buffer (100 mM Tris-HCl, pH 7.5, 12.5 mM EDTA, $150 \mathrm{mM} \mathrm{NaCl}, 1 \% \mathrm{SDS}$ ). After $30 \mathrm{~min}$ at $37^{\circ} \mathrm{C}$, RNA was recovered by ethanol precipitation. The labeling efficiency was measured by DEAE filter absorption (Stayton and Kornberg 1983). For the subsequent RNase A/T1-digestion, the following amounts were used: $100,000 \mathrm{cpm}$ of labeled poly(A) or L3pre- $\mathrm{A}_{105} ; 500,000 \mathrm{cpm}$ of steady-state RNA or rRNA; the entire $3^{\prime}$ labeling reaction for $4 \mathrm{~s} \mathrm{U}$-labeled RNA. Digestion was performed for $45 \mathrm{~min}$ at $30^{\circ} \mathrm{C}$ in $80 \mu \mathrm{L}$ containing $10 \mathrm{mM}$ Tris- $\mathrm{HCl}, \mathrm{pH} 8.0,200 \mathrm{mM} \mathrm{NaCl}, 15 \mu \mathrm{g}$ total yeast RNA (as the carrier), $2 \mu \mathrm{g}$ RNase A, and $500 \mathrm{U}$ RNase T1 (Sigma-Aldrich). The reactions were stopped as described above. After ethanol precipitation, samples were analyzed on an $8.3 \mathrm{M}$ urea/ $8 \%$ polyacrylamide gel. Since equal amounts of radioactivity were used for the analysis of the steady-state RNA, differences in the yield of RNA do not show up. For the pulse-labeled RNA, differences in intensity reflect both differences in cell growth and different RNA yields through the extended purification and analysis procedure. The data do not permit a conclusion whether the rate of mRNA synthesis is affected by the PABPN1 knockdown.

For RT-qPCR, 25\% of the purified 4sU-labeled RNA was reversetranscribed with random hexanucleotide primers as previously described (Schönemann et al. 2014). QPCRs were performed as triplicates in a 96-well format with the Light-Cycler 480 SYBR Green I Master Mix (Roche) in a Light-Cycler 480 II instrument (Roche) at an annealing temperature of $60^{\circ} \mathrm{C}$ for all primer pairs (Supplemental Table S1). Correct sizes of PCR products were verified. $C_{\mathrm{p}}$ values were calculated by the software provided by Roche. Relative quantification of mRNA/pre-mRNA levels was done by the $\Delta \Delta C_{\mathrm{p}}$ method (Livak and Schmittgen 2001). As a control, mock biotinylations and affinity purifications were carried out with RNA that had not been 4 sU-labeled. Subsequent RT-qPCR resulted in $C_{\mathrm{p}}$ values at least 9.5-fold lower than with pulse-labeled RNA (data not shown). Thus, background binding of RNA to the streptavidin beads was negligible.

\section{Western blotting}

For the quantitative analysis of PABPN1 after siRNA treatment, cells were washed twice with ice-cold PBS, scraped from the plate, and lysed in $50 \mathrm{mM}$ Tris- $\mathrm{HCl}$, pH 7.4, $300 \mathrm{mM} \mathrm{NaCl}, 1 \%$ SDS, $1 \mathrm{mM}$ dithiothreitol, $2 \mathrm{mg} / \mathrm{L}$ leupeptin, $1 \mathrm{mg} / \mathrm{L}$ pepstatin, $0.4 \mathrm{mM}$ PMSF. Protein amounts were quantified with the Bradford reagent RotiQuant (Roth) and compared to BSA standards. Rabbit antibody against bovine PABPN1 has been previously described (Krause et al. 1994). Monoclonal antibody against human vinculin was from Sigma (\# V9131). Secondary fluorescent donkey antibodies anti-rabbit IRDye800CW (\# 926-32213) and anti-mouse IRDye680 (\# 926-32222) were from LI-COR Biosciences. Anti-PABPN1 was used in a 1:1000 and anti-vinculin in a 1:2000 dilution in $1.25 \%$ milk powder in TN-Tween. Western blotting and analysis was done as previously described (Schönemann et al. 2014).

\section{Nucleotide analyses}

The extraction and analysis of nucleotides was performed as previously described (Gebelein et al. 1992) with minor modifications.
HEK293 cells were treated with siRNAs as above, and cells from two $10-\mathrm{cm}$ dishes (50\%-90\% confluent) were trypsinized and harvested by centrifugation for $3 \mathrm{~min}$ at $250 \mathrm{~g}$ and $4^{\circ} \mathrm{C}$. The cell pellet was washed twice with ice-cold PBS. One-quarter of the cells was used for total protein preparation, and the knockdown efficiency was determined by Western blotting. The wet weight of the remaining cells was determined, and 5 volumes of ice-cold perchloric acid $(0.33 \mathrm{M})$ were added. If necessary, the cells were stored in liquid nitrogen for up to $1 \mathrm{~h}$ and then thawed, homogenized mechanically, and incubated on ice for $20 \mathrm{~min}$. The lysate was neutralized by the addition of $5 / 3$ volumes (based on the pellet wet weight) of $1 \mathrm{M}$ potassium carbonate. The sample was gently mixed, again incubated on ice for $20 \mathrm{~min}$ and centrifuged for $20 \mathrm{~min}$ at $20,000 \mathrm{~g}$ and $4^{\circ} \mathrm{C}$. The clear supernatant was stored at $-80^{\circ} \mathrm{C}$. Immediately before HPLC analysis, samples were again centrifuged under the same conditions. At least $50 \mu \mathrm{L}$ of each sample was loaded onto a reversed phase column (SuperCosil LC C18, $15 \mathrm{~cm} \times 4.6 \mathrm{~mm} \times 3 \mu \mathrm{m}$ ) installed in a LaChrom 7000 HPLC system with a diode array detector, and nucleotides were separated by ion pair reversed phase chromatography. Spectra of the eluate (200-400 nm) were continuously recorded. Nucleotides were identified by their retention times (compared with standard substances) and their characteristic absorption spectra. Nucleotide concentrations of the experimental samples were determined by their peak areas against standard curves of known concentrations. Concentrations refer to the volume of the wet cell pellet. The energy charge was calculated as previously described (Berg et al. 2015).

\section{Protein expression and purification}

The S. pombe Pab2 open reading frame (UniProtKB/Swiss-Prot number: O14327) was amplified with Pwo DNA polymerase by nested PCR from a cDNA library kindly provided by Norbert Käufer, University of Braunschweig. The second primer pair introduced flanking NdeI and BamHI sites, which were used for temporary cloning into NdeI/BamHI opened pGM (Martin and Keller 1996). An Ncol/BamHI fragment coding for an N-terminally histagged Pab2 was excised and cloned into matching restriction sites of pET19b (Novagen), thereby replacing the original his-tag with an MAHHHHHH-tag. The correct sequence of this and the other expression plasmids was confirmed. Expression of Pab2 was performed in BL21(DE3) pUBS522 (Brinkmann et al. 1989) in M9 medium by addition of $0.4 \mathrm{mM}$ IPTG and further incubation for $3 \mathrm{~h}$ at $37^{\circ} \mathrm{C}$. The protein was purified on a NiNTA resin (QIAGEN), followed by chromatography on Resource S (GE Healthcare). For use in polyadenylation assays, $\mathrm{Pab} 2$ was concentrated in a Centricon-10 ultrafiltration device (Merck Millipore). The identity of the protein, with the N-terminal methionine cleaved off, was confirmed by mass spectrometry.

The plasmid pQE9-PlaI expressing $S$. pombe poly(A) polymerase (Ohnacker et al. 1996) was a gift from Walter Keller's laboratory (Biozentrum Basel). Bacterial expression of the his-tagged protein and affinity purification was done as previously described (Ohnacker et al. 1996) with minor variations. Resource Q chromatography (GE Healthcare) was used as a second purification step. The identity of PlaI was confirmed by MS analysis, and the main contaminating protein of $60 \mathrm{kDa}$ was identified as pyruvate kinase II.

An expression plasmid for $S$. cerevisiae poly(A) polymerase (pGM-yPAP1) was also obtained from Walter Keller's laboratory. The his-tagged protein was expressed in BL21(DE3) pLysS 
(Novagen) for $5 \mathrm{~h}$ at $37^{\circ} \mathrm{C}$ after induction with $0.4 \mathrm{mM}$ IPTG. Purification was done by IMAC followed by a MonoS column (GE Healthcare). Bovine His-PAPa and His-PABPN1 were as previously described (Kerwitz et al. 2003; Kühn et al. 2009).

Protein concentrations were determined spectrophotometrically at $280 \mathrm{~nm}$ using calculated extinction coefficients (PABPN1: 18,730 $\mathrm{M}^{-1} \mathrm{~cm}^{-1}$; Pab2: $6640 \mathrm{M}^{-1} \mathrm{~cm}^{-1}$; PAPa: 67,530 $\mathrm{M}^{-1} \mathrm{~cm}^{-1}$; PlaI: 78,100 $\mathrm{M}^{-1} \mathrm{~cm}^{-1}$; S. cerevisiae PAP: $62,550 \mathrm{M}^{-1} \mathrm{~cm}^{-1}$ ). Concentrations of $\mathrm{Pab} 2$ in individual column fractions used for RNA binding assays were measured by comparison to BSA standards in a Coomassie-stained SDS-gel and by Bradford assay (Roth) because of the low extinction coefficient of the protein.

\section{In vitro assays}

Nonspecific poly(A) polymerase activities were determined in the presence of $\mathrm{MnCl}_{2}$ by elongation of synthetic $\mathrm{A}_{14}$ primers (IBA Lifesciences) with $\left[a-{ }^{32} \mathrm{P}\right]-\mathrm{ATP}$. Two different reaction conditions were applied (Lingner et al. 1991; Wahle 1991b). Reactions were stopped after $30 \mathrm{~min}$ by addition of $10 \mathrm{mM}$ EDTA. An aliquot of the reaction mix was absorbed on pretreated DEAE filter paper and washed (Stayton and Kornberg 1983) until an empty filter included as a control was devoid of radioactivity.

The stimulation of poly(A) polymerases by poly(A) binding proteins was measured as follows: $400 \mathrm{fmol}$ ( $3^{\prime}$ ends) of size-fractionated poly(A) $(\sim 105 \mathrm{nt})$ were incubated for $32 \mathrm{~min}$ at $32^{\circ} \mathrm{C}$ with $200 \mathrm{fmol}$ polymerase (PAPa or PlaI) and increasing amounts of poly $(\mathrm{A})$ binding protein (PABPN1 or Pab2) in SPAB buffer (25 mM Tris-HCl, $\mathrm{pH}$ 8.0, $50 \mathrm{mM} \mathrm{KCl}, 2 \mathrm{mM} \mathrm{MgCl}_{2}, 10 \%$ glycerol, $0.05 \mathrm{mM}$ EDTA, $1 \mathrm{mM}$ DTT, $0.4 \mathrm{~g} / \mathrm{L}$ methylated BSA, 0.01\% NP40, 2\% PEG 6000, $8 \mathrm{U}$ Ribolock RNase Inhibitor, and $\left.0.5 \mathrm{mM}\left[\alpha^{-}{ }^{32} \mathrm{P}\right]-\mathrm{ATP}\right)$ in a total volume of $20 \mu \mathrm{L}$. Because Plal activity was found to be salt-sensitive, $\mathrm{KCl}$ introduced with varying amounts of poly(A) binding proteins was accommodated by use of an SPAB stock lacking salt and separate addition of the required amounts of $\mathrm{KCl}$. In contrast to the standard reaction conditions previously used for PAPa and PABPN1, tRNA was omitted because it competed with poly(A) for binding to Pab2. Incorporation of ATP was measured as above. Under these conditions, PAPa by itself incorporated 5.4 pmol ATP, whereas PlaI by itself incorporated $\sim 35$ pmol ATP. For the determination of steady-state kinetic parameters, synthetic $\mathrm{A}_{45}$ (TIB MOLBIOL, Berlin, Germany) was used as a primer, and $K_{\mathrm{M}}$ and $V_{\max }$ were estimated from Lineweaver-Burk plots.

For electrophoretic mobility shift assays, $80 \mathrm{fmol}$ ( $3^{\prime}$ ends) of $5^{\prime}$-labeled $A_{100}$ were incubated in a $20 \mu \mathrm{L}$ volume for $30 \mathrm{~min}$ at room temperature with increasing amounts of Pab2 in SPAB buffer (see above). One half of each binding reaction was applied to a composite native gel as previously described (Rüegsegger et al. 1996).

Filter-binding experiments were carried out as previously described (Kühn et al. 2003) with the following modifications: Binding was performed in $50 \mu \mathrm{L}$ FBK50 buffer $(50 \mathrm{mM}$ Tris- $\mathrm{HCl}$, pH 8.0, $50 \mathrm{mM} \mathrm{KCl}, 10 \%$ glycerol, $1 \mathrm{mM}$ EDTA, $1 \mathrm{mM}$ DTT, 0.2 g/L methylated BSA, $0.01 \%$ NP40) containing either $10 \mathrm{fmol}\left(3^{\prime}\right.$ ends) $\mathrm{A}_{14}$ or $\sim 1.5 \mathrm{fmol}$ of $\mathrm{A}_{100}$ and increasing amounts of poly(A) binding protein. After $20 \mathrm{~min}$ at room temperature, $45 \mu \mathrm{L}$ of each binding reaction was applied to nitrocellulose filters. Competition experiments were done under the same conditions but using 15 fmol radiolabeled $\mathrm{A}_{100}$ and $1 \mathrm{nM}$ Pab2 and increasing amounts of competitors: E. coli rRNA, E. coli tRNA, poly(C) or poly(A) (all from Roche) in FBK50.

\section{SUPPLEMENTAL MATERIAL}

Supplemental material is available for this article.

\section{ACKNOWLEDGMENTS}

We are grateful to Gudrun Scholz for technical assistance, Yvonne Kerwitz for cloning of S. pombe Pab2, Babette Pinkwart and Katharina Fronz for initial experiments, Walter Keller for plasmids, Norbert Käufer for a gift of S. pombe cDNA, Angelika Schierhorn for MS analyses, and Mike Schutkowski for permission to use his HPLC. This work was supported by funding from the Deutsche Forschungsgemeinschaft (to E.W.).

Received April 20, 2016; accepted January 4, 2017.

\section{REFERENCES}

Apponi LH, Leung SW, Williams KR, Valentini SR, Corbett AH, Pavlath GK. 2010. Loss of nuclear poly(A)-binding protein 1 causes defects in myogenesis and mRNA biogenesis. Hum Mol Genet 19: 1058-1065.

Bear DG, Fomproix N, Soop T, Björkroth B, Masich S, Daneholt B. 2003. Nuclear poly(A)-binding protein PABPN1 is associated with RNA polymerase II during transcription and accompanies the released transcript to the nuclear pore. Exp Cell Res 286: 332-344.

Beaulieu YB, Kleinman CL, Landry-Voyer AM, Majewski J, Bachand F. 2012. Polyadenylation-dependent control of long noncoding RNA expression by the poly(A)-binding protein nuclear 1. PLoS Genet 8: e1003078.

Benoit B, Mitou G, Chartier A, Temme C, Zaessinger S, Wahle E, Busseau I, Simonelig M. 2005. An essential cytoplasmic function for the nuclear poly (A) binding protein, $\mathrm{PABP} 2$, in poly(A) tail length control and early development in Drosophila. Dev Cell 9: 511-522.

Berg JM, Tymoczko JL, Gatto GJJ, Stryer L. 2015. Biochemistry. W. H. Freeman and Company, New York.

Brawerman G. 1981. The role of the poly(A) sequence in mammalian messenger RNA. CRC Crit Rev Biochem 10: 1-38.

Bresson SM, Conrad NK. 2013. The human nuclear poly(A)-binding protein promotes RNA hyperadenylation and decay. PLoS Genet 9: e1003893.

Bresson SM, Hunter OV, Hunter AC, Conrad NK. 2015. Canonical poly(A) polymerase activity promotes the decay of a wide variety of mammalian nuclear RNAs. PLoS Genet 11: e1005610.

Brinkmann U, Mattes RE, Buckel P. 1989. High-level expression of recombinant genes in Escherichia coli is dependent on the availability of the dnaY gene product. Gene 85: 109-114.

Calado A, Kutay U, Kühn U, Wahle E, Carmo-Fonseca M. 2000. Deciphering the cellular pathway for transport of poly(A)-binding protein II. RNA 6: 245-256.

Chang H, Lim J, Ha M, Kim VN. 2014. TAIL-seq: genome-wide determination of poly(A) tail length and $3^{\prime}$ end modifications. Mol Cell 53: 1044-1052.

Chartier A, Klein P, Pierson S, Barbezier N, Gidaro T, Casas F, Carberry S, Dowling P, Maynadier L, Bellec M, et al. 2015. Mitochondrial dysfunction reveals the role of mRNA poly(A) tail regulation in oculopharyngeal muscular dystrophy pathogenesis. PLoS Genet 11: e1005092.

Chen CYA, Shyu AB. 1994. Selective degradation of early-response-gene messenger-RNAs: functional analyses of sequence features of the AU-rich elements. Mol Cell Biol 14: 8471-8482.

Chomczynski P, Mackey K. 1995. Short technical reports. Modification of the TRI reagent procedure for isolation of RNA from polysaccharide- and proteoglycan-rich sources. Biotechniques 19: 942-945.

Cosson B, Braun F, Paillard L, Blackshear P, Beverley Osborne H. 2004. Identification of a novel Xenopus laevis poly(A) binding protein. Biol Cell 96: 519-527. 
de Klerk E, Venema A, Anvar SY, Goeman JJ, Hu O, Trollet C, Dickson G, den Dunnen JT, van der Maarel SM, Raz V, et al. 2012. Poly(A) binding protein nuclear 1 levels affect alternative polyadenylation. Nucleic Acids Res 40: 9089-9101.

Dolken L, Ruzsics Z, Rädle B, Friedel CC, Zimmer R, Mages J, Hoffmann R, Dickinson P, Forster T, Ghazal P, et al. 2008. High-resolution gene expression profiling for simultaneous kinetic parameter analysis of RNA synthesis and decay. RNA 14: 1959-1972.

Ge H, Zhou D, Tong S, Gao Y, Teng M, Niu L. 2008. Crystal structure and possible dimerization of the single RRM of human PABPN1. Proteins 71: 1539-1545.

Gebelein M, Merdes G, Berger MR. 1992. Nucleotide preparation from cells and determination of nucleotides by ion-pair high-performance liquid chromatography. J Chromatogr 577: 146-150.

Good PJ, Abler L, Herring D, Sheets MD. 2004. Xenopus embryonic poly (A) binding protein 2 (ePABP2) defines a new family of cytoplasmic poly(A) binding proteins expressed during the early stages of vertebrate development. Genesis 38: 166-175.

Jenal M, Elkon R, Loayza-Puch F, van Haaften G, Kühn U, Menzies FM, Oude Vrielink JA, Bos AJ, Drost J, Rooijers K, et al. 2012. The poly (A)-binding protein nuclear 1 suppresses alternative cleavage and polyadenylation sites. Cell 149: 538-553.

Kerwitz Y, Kühn U, Lilie H, Knoth A, Scheuermann T, Friedrich H, Schwarz E, Wahle E. 2003. Stimulation of poly(A) polymerase through a direct interaction with the nuclear poly(A) binding protein allosterically regulated by RNA. EMBO J 22: 3705-3714.

Krause S, Fakan S, Weis K, Wahle E. 1994. Immunodetection of poly(A) binding protein II in the cell nucleus. Exp Cell Res 214: 75-82.

Kühn U, Wahle E. 2004. Structure and function of poly(A) binding proteins. Biochim Biophys Acta 1678: 67-84.

Kühn U, Nemeth A, Meyer S, Wahle E. 2003. The RNA binding domains of the nuclear poly(A)-binding protein. J Biol Chem 278: $16916-16925$

Kühn U, Gündel M, Knoth A, Kerwitz Y, Rüdel S, Wahle E. 2009. Poly (A) tail length is controlled by the nuclear poly(A)-binding protein regulating the interaction between poly(A) polymerase and the cleavage and polyadenylation specificity factor. J Biol Chem 284: 22803-22814.

Lemay JF, D'Amours A, Lemieux C, Lackner DH, St-Sauveur VG, Bähler J, Bachand F. 2010. The nuclear poly(A)-binding protein interacts with the exosome to promote synthesis of noncoding small nucleolar RNAs. Mol Cell 37: 34-45.

Lemieux C, Bachand F. 2009. Cotranscriptional recruitment of the nuclear poly(A)-binding protein Pab2 to nascent transcripts and association with translating mRNPs. Nucleic Acids Res 37: 3418-3430.

Lemieux C, Marguerat S, Lafontaine J, Barbezier N, Bähler J, Bachand F. 2011. A pre-mRNA degradation pathway that selectively targets intron-containing genes requires the nuclear poly(A)-binding protein. Mol Cell 44: 108-119.

Li W, You B, Hoque M, Zheng D, Luo W, Ji Z, Park JY, Gunderson SI, Kalsotra A, Manley JL, et al. 2015. Systematic profiling of poly(A) transcripts modulated by core $3^{\prime}$ end processing and splicing factors reveals regulatory rules of alternative cleavage and polyadenylation. PLoS Genet 11: e1005166.

Lingner J, Radtke I, Wahle E, Keller W. 1991. Purification and characterization of poly(A) polymerase from Saccharomyces cerevisiae. J Biol Chem 266: 8741-8746.

Livak KJ, Schmittgen TD. 2001. Analysis of relative gene expression data using real-time quantitative PCR and the $2^{-\Delta \Delta C_{T}}$ method. Methods 25: 402-408.

Mangus DA, Evans MC, Jacobson A. 2003. Poly(A)-binding proteins: multifunctional scaffolds for the post-transcriptional control of gene expression. Genome Biol 4: 223.

Martin G, Keller W. 1996. Mutational analysis of mammalian poly(A) polymerase identifies a region for primer binding and a catalytic domain, homologous to the family $\mathrm{X}$ polymerases, and to other nucleotidyl transferases. EMBO J 15: 2593-2603.

Muniz L, Davidson L, West S. 2015. Poly(A) polymerase and the nuclear poly(A) binding protein, PABPN1, coordinate the splicing and deg- radation of a subset of human pre-mRNAs. Mol Cell Biol 35: 2218-2230.

Nemeth A, Krause S, Blank D, Jenny A, Jenö P, Lustig A, Wahle E. 1995. Isolation of genomic and cDNA clones encoding bovine poly(A) binding protein II. Nucleic Acids Res 23: 4034-4041.

Nguyen D, Grenier St-Sauveur V, Bergeron D, Dupuis-Sandoval F, Scott MS, Bachand F. 2015. A polyadenylation-dependent $3^{\prime}$ end maturation pathway is required for the synthesis of the human telomerase RNA. Cell Rep 13: 2244-2257.

Ohnacker M, Minvielle-Sebastia L, Keller W. 1996. The Schizosaccharomyces pombe pla1 gene encodes a poly(A) polymerase and can functionally replace its Saccharomyces cerevisiae homologue. Nucleic Acids Res 24: 2585-2591.

Perreault A, Lemieux C, Bachand F. 2007. Regulation of the nuclear poly (A)-binding protein by arginine methylation in fission yeast. J Biol Chem 282: 7552-7562.

Rüegsegger U, Beyer K, Keller W. 1996. Purification and characterization of human cleavage factor I involved in the $3^{\prime}$ end processing of messenger RNA precursors. J Biol Chem 271: 6107-6113.

Sawicki SG, Jelinek W, Darnell JE. 1977. 3'-Terminal addition to HeLa cell nuclear and cytoplasmic poly(A). J Mol Biol 113: 219-235.

Schiavi SC, Wellington CL, Shyu AB, Chen CYA, Greenberg ME, Belasco JG. 1994. Multiple elements in the c-fos protein-coding region facilitate messenger-RNA deadenylation and decay by a mechanism coupled to translation. J Biol Chem 269: 3441-3448.

Schönemann L, Kühn U, Martin G, Schäfer P, Gruber AR, Keller W, Zavolan M, Wahle E. 2014. Reconstitution of CPSF active in polyadenylation: recognition of the polyadenylation signal by WDR33. Genes Dev 28: 2381-2393.

Shyu AB, Belasco JG, Greenberg ME. 1991. Two distinct destabilizing elements in the c-fos message trigger deadenylation as a first step in rapid mRNA decay. Genes Dev 5: 221-231.

Smith JJ, Rücknagel KP, Schierhorn A, Tang J, Nemeth A, Linder M, Herschman HR, Wahle E. 1999. Unusual sites of arginine methylation in poly(A)-binding protein II and in vitro methylation by protein arginine methyltransferases PRMT1 and PRMT3. J Biol Chem 274: 13229-13234.

Song J, McGivern JV, Nichols KW, Markley JL, Sheets MD. 2008. Structural basis for RNA recognition by a type II poly(A)-binding protein. Proc Natl Acad Sci 105: 15317-15322.

St-Andre O, Lemieux C, Perreault A, Lackner DH, Bahler J, Bachand F. 2010. Negative regulation of meiotic gene expression by the nuclear poly(a)-binding protein in fission yeast. $J$ Biol Chem 285: 27859-27868.

Stayton M, Kornberg A. 1983. Complexes of Escherichia coli primase with the replication origin of G4 phage DNA. J Biol Chem 258: 13205-13212.

Subtelny AO, Eichhorn SW, Chen GR, Sive H, Bartel DP. 2014. Poly(A)tail profiling reveals an embryonic switch in translational control. Nature 508: 66-71.

Wahle E. 1991a. A novel poly(A)-binding protein acts as a specificity factor in the second phase of messenger RNA polyadenylation. Cell 66: 759-768.

Wahle E. 1991b. Purification and characterisation of a mammalian polyadenylate polymerase involved in the $3^{\prime}$ end processing of messenger RNA precursors. J Biol Chem 226: 3131-3139.

Wahle E. 1995. Poly(A) tail length control is caused by termination of processive synthesis. J Biol Chem 270: 2800-2808.

Wigington CP, Williams KR, Meers MP, Bassell GJ, Corbett AH. 2014. Poly(A) RNA-binding proteins and polyadenosine RNA: new members and novel functions. Wiley Interdiscip Rev RNA 5: 601-622.

Winstall E, Sadowski M, Kühn U, Wahle E, Sachs AB. 2000. The Saccharomyces cerevisiae RNA-binding protein Rbp29 functions in cytoplasmic mRNA metabolism. J Biol Chem 275: 21817-21826.

Yamanaka S, Yamashita A, Harigaya Y, Iwata R, Yamamoto M. 2010. Importance of polyadenylation in the selective elimination of meiotic mRNAs in growing S. pombe cells. EMBO J 29: 2173-2181. 

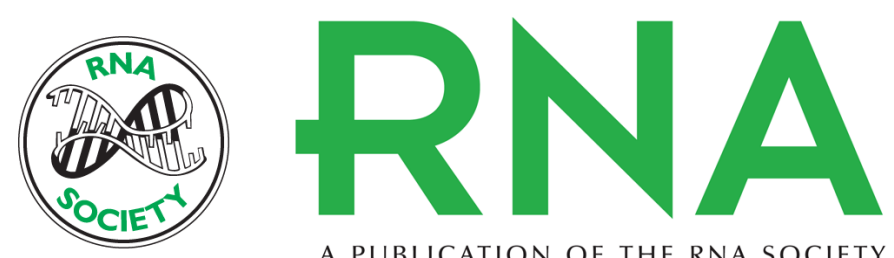

A PUBLICATION OF THE RNA SOCIETY

\section{The nuclear poly(A) binding protein of mammals, but not of fission yeast, participates in mRNA polyadenylation}

Uwe Kühn, Juliane Buschmann and Elmar Wahle

RNA 2017 23: 473-482 originally published online January 17, 2017

Access the most recent version at doi:10.1261/rna.057026.116

\section{Supplemental http://rnajournal.cshlp.org/content/suppl/2017/01/17/rna.057026.116.DC1 \\ Material}

References This article cites 54 articles, 20 of which can be accessed free at: http://rnajournal.cshlp.org/content/23/4/473.full.html\#ref-list-1

Creative This article is distributed exclusively by the RNA Society for the first 12 months after the Commons License full-issue publication date (see http://rnajournal.cshlp.org/site/misc/terms.xhtml). After 12 months, it is available under a Creative Commons License (Attribution-NonCommercial 4.0 International), as described at http://creativecommons.org/licenses/by-nc/4.0/.
Email Alerting Receive free email alerts when new articles cite this article - sign up in the box at the Service top right corner of the article or click here.

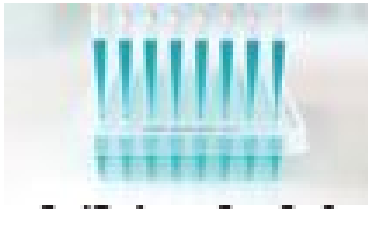

\section{Providing Precise Solutions for} your research.

To subscribe to $R N A$ go to:

http://rnajournal.cshlp.org/subscriptions 